\title{
Anhaltende komplexe Trauerreaktion - ein neues Krankheitsbild?
}

\author{
Jana Steinig, Anette Kersting
}

Menschen trauern sehr unterschiedlich. Wird der normale Trauerprozess nicht abgeschlossen, kann es zu einer anhaltenden komplexen Trauerreaktion kommen. Diese bedarf einer spezifischen psychotherapeutischen Behandlung.

\section{Einleitung}

Trauer ist ein universelles Phänomen, das dazu dient, sich an die neue, grundlegend und unwiderruflich veränderte Lebenswirklichkeit anzupassen, die durch den schmerzhaften Verlust eines geliebten Menschen entstanden ist. Trauer ist ein ubiquitärer Vorgang, mit dem Verlust und Tod, die in das eigene Leben eingebrochen sind und es oftmals in ein "Davor" und ein „Danach“ einteilen, integriert und akzeptiert werden können.

Trauer ist die normale emotionale Reaktion auf den Verlust eines nahestehenden Menschen und kein pathologisches Phänomen.

Der Trauerprozess selbst ist somit eine Art Übergangszustand, zwischen dem Wissen, dass ein geliebter Mensch gestorben ist und der Akzeptanz dieses Verlustes. Durch den Prozess des Trauerns wird es möglich, in einem Wechsel zwischen der rückwärtsgerichteten Beschäftigung mit dem Verlust und einer zukunftsorientierten Perspektive auf kommende Aufgaben,

- die Beziehung zu der verstorbenen Person neu zu ordnen,

- sich zu verabschieden und

- allmählich in den Alltag und das aktuelle Leben zurückzufinden.
Nicht selten führt die Auseinandersetzung mit dem Verlust und der damit verbundenen Trauer zu einer Beschäftigung mit dem eigenen Selbstkonzept und dem eigenen Selbstverständnis, zu einer Stärkung der Bewältigungsfähigkeit sowie zu einem neuen Blick auf die Welt, das Leben und den Tod. Der Trauerprozess kann somit auch als Entwicklungsprozess verstanden werden [1]. Es kann jedoch auch zu Störungen in diesem Prozess kommen, die dazu führen, dass die Trauersymptomatik persistiert und in eine pathologische Trauerreaktion mündet.

Der vorliegende Artikel befasst sich zunächst mit dem normalen Trauerprozess und möglichen psychischen und physischen Folgen eines Verlustes. Im Anschluss daran wird das klinische Erscheinungsbild der anhaltenden komplexen Trauerreaktion beschrieben, es werden diagnostische und differenzialdiagnostische Kriterien und mögliche Risikofaktoren diskutiert. Konzepte zur Behandlung der anhaltenden komplexen Trauerreaktion werden am Ende des Artikels dargestellt. 


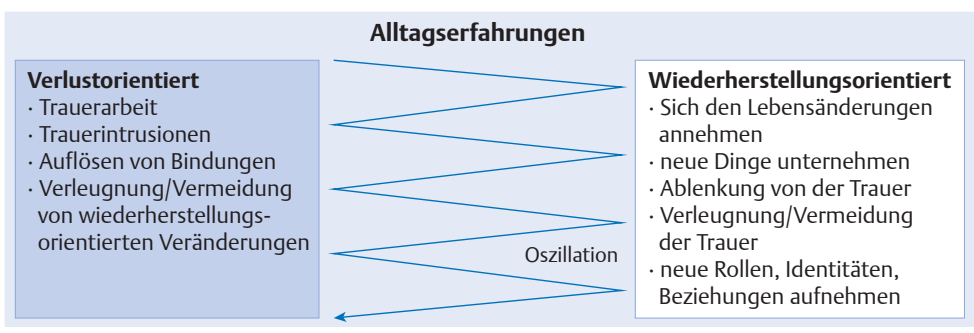

Abb.1 Das duale Prozessmodell der Trauerbewältigung von Ströbe und Schut [3].

\section{Der Trauerprozess}

\section{Phasenmodelle}

In der klinischen Literatur werden verschiedene Phasenmodelle beschrieben, die den Trauerprozess als Durchleben verschiedener Trauerphasen abbilden, z. B.

- Nicht-wahrhaben-Wollen

- Zorn und Ärger

- Verhandeln

- Depression

- Zustimmung [2]

an deren Ende die Verarbeitung des Verlustes steht..

Phasenmodelle sind weit verbreitet und bieten Betroffenen und Behandelnden eine hilfreiche Möglichkeit, einen Einblick in Trauerprozesse zu erhalten. Sie entsprechen der Vorstellung, es bedürfe einer spezifischen „Trauerarbeit“ in Form des Durcharbeitens verschiedener aufeinanderfolgender Phasen, um über einen Verlust hinwegzukommen. Zu bedenken ist allerdings, dass Trauerprozesse heterogen und individuell verlaufen und Phasenmodelle dies nur unzureichend berücksichtigen können. Zudem müssen auch kulturelle Einflussfaktoren auf den Trauerverlauf beachtet werden. Dies erschwert die Definition eines normalen Trauerprozesses und die Abgrenzung normaler Trauer von einem pathologischen Verlauf. So wird die Frage, ab welcher Dauer und welcher Intensität Trauersymptome krankheitswertig sind und als psychische Störung klassifiziert werden sollten, ebenso kontrovers diskutiert wie die Frage nach dem Ziel der Trauerarbeit: Steht die Loslösung von der verstorbenen Person im Vordergrund oder in gleichem Maße auch die Integration der verstorbenen Person in das Leben der Hinterbliebenen?

Die Vielfalt der Trauerprozesse erschwert eine Abgrenzung normaler und pathologischer Trauerverläufe.

\section{Tipp für die Praxis}

Auch wenn verschiedene Phasenmodelle zur Trauer existieren, handelt es sich bei der Trauer um einen nahestehenden Menschen immer um einen individuellen Prozess.

\section{Duales Prozessmodell}

Bei dem dualen Prozessmodell von Ströbe und Schut steht im Zentrum nicht primär und ausschließlich die Verarbeitung der Trauer und die Loslösung von dem Verstorbenen, sondern parallel dazu gleichwertig auch die Bewältigung der neuen veränderten Situation ohne die verstorbene Person (Abb. 1) [3]. Dementsprechend betont dieses Modell das kontinuierliche Oszillieren zwischen 2 Foki:

- die verlustorientierte Bewältigung (z.B. Trauerarbeit, Lösen von emotionalen Bindungen zu der verstorbenen Person) und

- die wiederherstellungsorientierte Bewältigung (z.B. Eingehen neuer Beziehungen, Aufnehmen neuer Aktivitäten).

Ziel dieses dynamischen Prozesses ist sowohl die Integration des Verlustes, wie auch die Orientierung auf neue Lebensziele.

Das duale Prozessmodel unterscheidet zwischen verlust- und wiederherstellungsorientierter Bewältigung und unterstreicht die Dynamik der Trauerbewältigung, die durch ein fortwährendes Oszillieren zwischen diesen beiden Verarbeitungsprozessen gekennzeichnet ist.

\section{Definition normaler Trauer}

Das Trauererleben nach dem Verlust eines nahestehenden Menschen ist durch das Auftreten von intensivem Trennungsschmerz, Sehnsucht, Traurigkeit, schmerzvollen aber auch positiven Erinnerungen an die verstorbene Person und gemeinsam Erlebtes gekennzeichnet. Die Intensität der Trauer nimmt im Verlauf der Zeit ab und es gelingt der trauernden Person, sich allmählich an die neuen veränderten Lebensumstände ohne die verstorbene Person anzupassen, den Blick wieder in die Zukunft zu richten und sich neuen Aufgaben, Aktivitäten und Beziehungen zuzuwenden. 


\section{Fallbeispiel 1}

\section{Normaler Trauerverlauf}

Herr X., 69 Jahre, verliert seine Frau nach einer langen schweren Krebserkrankung. Aufgrund der langen Krankheit hat sich Herr X. bereits schrittweise mit dem nahenden Tod seiner Frau auseinandergesetzt. Beide haben in vielen Gesprächen auf das gemeinsame Leben zurückgeschaut und auch darüber gesprochen, wie es für Herrn X. allein weitergehen könnte. Dennoch versetzt ihn der Tod seiner Frau in einen Schockzustand. Er fühlt sich wie betäubt, kann keinen klaren Gedanken fassen und ist nicht in der Lage, den Tod seiner Frau zu realisieren. Nach ein paar Tagen erlebt er heftige Emotionen wie eine intensive Trauer, die er als körperlichen Schmerz spürt, Zorn, Verzweiflung und eine intensive Sehnsucht nach seiner Frau. Bei der Beisetzung seiner Frau erlebt er seine beiden Kinder als Halt, beide leben jedoch mit ihren Familien in größerer Entfernung, sodass häufigere Besuche in den kommenden Wochen nicht möglich sind. Herr $X$. denkt häufig und intensiv an seine verstorbene Frau und ihr gemeinsames vergangenes Leben. Viele Dinge, Orte und Handlungen in seinem Alltag erinnern ihn an sie und seine Gedanken und Träume sind stark von dem Andenken an seine Frau dominiert. Oft geht er auf den Friedhof und hält am Grab seiner Frau Zwiegespräche mit ihr. Da in ihrer Ehe immer sie diejenige war, die sich um die sozialen Kontakte kümmerte, fällt es Herrn X. sehr schwer, auf andere Menschen zuzugehen. Ermutigt durch seine Kinder knüpft er nach einiger Zeit jedoch Kontakt zu seiner Kirchengemeinde und schließt sich einem Lesekreis für ältere Menschen an. Einige Monate nach dem Tod seiner Frau berichtet Herr X. weiterhin von Phasen ausgeprägten Trauerschmerzes. Gleichzeitig werden die Phasen, in denen Herr X. nicht von Gedanken an seine Frau beherrscht wird, länger und es gelingt ihm zunehmend besser, sich in seinem neuen Alltag zurechtzufinden.

\section{Fehlende und verzögerte Trauer}

Wie oben schon erwähnt, ist eine allgemeingültige Normierung der normalen Trauer schwierig. So weisen Studien darauf hin, dass auch eine fehlende oder eine verzögerte Trauerreaktion möglich ist und es sich dabei um weitere Variationen des Bewältigungsprozesses handelt [4].

Fazit. Zusammenfassend ist festzuhalten, dass sich ein normaler, nicht pathologischer Trauerprozess nur schwer normieren lässt, da sowohl die Intensität als auch die Dauer individuell unterschiedlich sein können.

Verschiedene Faktoren, wie die Todesart oder die Todesumstände, aber auch die Beziehung zu der verstorbenen Person beeinflussen die Verarbeitung des Verlustes.

\section{Psychische und physische Folgen von Verlusten}

Die Trauer um einen nahestehenden Menschen tritt oftmals gemeinsam mit Symptomen von Angst, Depression oder Substanzmissbrauch auf. Diese Symptome sind häufig ein Teil des Trauerprozesses, können allerdings auch in eine eigenständige manifeste psychische Erkrankung (z.B. Angststörung oder Depression) münden. Eine eigene Studie zeigte, dass fast 17\% der Frauen, die ihr Kind in der Spätschwangerschaft aufgrund fetaler Fehlbildungen verloren, 14 Monate nach dem Verlust an einer manifesten psychischen Störung litten. Während 2 Wochen nach dem Verlust affektive Erkrankungen, Angststörungen, Essstörungen und akute Belastungsreaktionen diagnostiziert wurden, waren es 14 Monate nach dem Verlust ausschließlich Depressionen und Angsterkrankungen [5].

Trauerprozesse können in psychische oder physische Erkrankungen münden.

Die Verarbeitung von Verlusten ist auch mit einem erhöhten Risiko für körperliche Erkrankungen verbunden. So wurde in Untersuchungen eine Veränderung verschiedener physiologischer Parameter gefunden, wie z.B. erhöhter Herzschlag und erhöhter Blutdruck, erhöhter Kortisolspiegel, Schlafstörungen und Veränderungen des Immunsystems. Auch Gewichtsverlust, Schmerzen und vermehrte allgemeine körperliche Beschwerden finden sich häufig bei Trauernden, ebenso wie ein erhöhtes Herzinfarkt- oder Stress-Kardiomyopathie-Risiko. Darüber hinaus ist der Tod eines nahen Angehörigen mit einem erhöhten Mortalitätsrisiko des Verbliebenen assoziiert. Dies gilt v. a. für die erste Zeit direkt nach dem Verlust, wobei andere Studien ein erhöhtes Risiko auch 6 Monate oder länger nach dem Verlusterleben beobachtet haben [6]. Tab. 1 fasst eine Vielzahl unterschiedlicher möglicher Symptome und Reaktionen auf einen Verlust zusammen. 


\section{Tabelle 1}

Mögliche Reaktionen auf einen Verlust (nach [6]).

\begin{tabular}{|c|c|}
\hline Reaktion & Symptome \\
\hline affektive Reaktionen & $\begin{array}{l}\text { - Depression, Verzweiflung, Niedergeschlagenheit, } \\
\text { - Achmerz } \\
\text { - Angst, Sorgen, Schrecken } \\
\text { - Schuldgefühle, Selbstvorwürfe, Selbstbeschuldigungen } \\
\text { - Anhedonie - Verlust von Freude } \\
\text { - Einsamkeit } \\
\text { - Sehnsucht, Verlangen } \\
\text { - Schock, Taubheit }\end{array}$ \\
\hline kognitive Reaktionen & $\begin{array}{l}\text { - beherrschende intrusive Gedanken an den Toten, } \\
\text { Grübeln } \\
\text { - Gefühl der Anwesenheit der verstorbenen Person } \\
\text { - Verdrängung, Verleugnung } \\
\text { - vermindertes Selbstbewusstsein } \\
\text { - Hilflosigkeit, Hoffnungslosigkeit } \\
\text { - Suizidgedanken } \\
\text { - Gefühl der Unwirklichkeit } \\
\text { - Gedächtnis- und Konzentrationsschwierigkeiten }\end{array}$ \\
\hline $\begin{array}{l}\text { verhaltensbezogene } \\
\text { Reaktionen }\end{array}$ & $\begin{array}{l}\text { - Agitation, Angespanntheit, Ruhelosigkeit } \\
\text { - Müdigkeit } \\
\text { - Überaktivität } \\
\text { - Suchen } \\
\text { - Weinen } \\
\text { - sozialer Rückzug }\end{array}$ \\
\hline $\begin{array}{l}\text { physiologische } \\
\text { Reaktionen }\end{array}$ & $\begin{array}{l}\text { - Appetitverlust } \\
\text { - Schlafstörungen } \\
\text { - Erschöpfung, Energielosigkeit } \\
\text { - somatische Beschwerden } \\
\text { - physische Beschwerden ähnlich denen der } \\
\text { verstorbenen Person } \\
\text { - Anfälligkeit für Krankheiten, Mortalität }\end{array}$ \\
\hline
\end{tabular}

\section{Anhaltende komplexe Trauer- reaktion}

Gelingt die Verarbeitung eines Verlustes nicht, kann der Trauerprozess in eine pathologische Trauerreaktion münden, die durch das Steckenbleiben bzw. Verharren im Trauerprozess gekennzeichnet ist. Sie unterscheidet sich von der normalen Trauer sowohl durch die Intensität als auch die Qualität und Dauer der Trauersymptome. Dadurch kommt es zu einer pathologischen Symptomatik und Einschränkungen in verschiedenen Lebensbereichen.

Begrifflichkeiten. Bis heute existieren verschiedene Begriffe zur Beschreibung einer pathologischen Trauerreaktion, was oftmals zu einer gewissen Verwirrung führt. So wird von pathologischer Trauer ebenso gesprochen, wie von prolongierter Trauer, traumatischer
Trauer oder komplizierter Trauer. Am ehesten etabliert hat sich bislang der Begriff der komplizierten Trauer (analog zu „Complicated Grief“ in der internationalen Literatur). Mit der diskutierten Einführung des klinischen Erscheinungsbilds der komplizierten Trauer in die diagnostischen Klassifikationssysteme (s. u.), wird der Begriff voraussichtlich durch „Anhaltende komplexe Trauerreaktion“ (DSM-5) bzw. „Prolongierte Trauerstörung“ (ICD-11) zunehmend abgelöst. Da bisherige Studien zumeist den Begriff der komplizierten Trauer benutzen, wird diese Bezeichnung im Folgenden ebenfalls zur Beschreibung pathologischer Trauerprozesse verwendet.

Für pathologische Trauerreaktionen gibt es zahlreiche Begriffe - im neu erschienenen DSM-5 wird der Begriff der „Anhaltenden komplexen Trauerreaktion" verwendet.

\section{Eigenständiges Krankheitsbild? In den vergangenen} Jahren wurde debattiert, ob das bislang als komplizierte Trauer bezeichnete klinische Erscheinungsbild überhaupt als eigenständiges Krankheitsbild zu betrachten ist oder nicht eher in ein bereits bestehendes (z. B. Depression, Posttraumatische Belastungsstörung) integriert werden sollte. Verschiedene Studien weisen jedoch darauf hin, dass es sich bei der komplizierten Trauer um ein abgrenzbares Phänomen handelt, das deutliche Unterschiede zu den bereits bestehenden Krankheitsbildern aufweist (s. auch Differenzialdiagnostik) [7-9].

\section{Klassifikationssysteme}

Obwohl sich die komplizierte Trauer von anderen Krankheitsbildern abgrenzen lässt, wurde ihre Aufnahme als eigenständige psychische Störung in die diagnostischen Klassifikationssysteme kontrovers diskutiert. Es existieren unterschiedliche Vorschläge zu den Diagnosekriterien [10-12].

DSM-5. In der aktuellen und überarbeiteten 5. Ausgabe des Diagnostic and Statistical Manual of Mental Disorders (DSM-5) wurde die komplizierte Trauer vor dem Hintergrund einer Vielzahl empirischer Studien erstmals aufgenommen, jedoch nicht als eigenständiges diagnostisches Kriterium, sondern in Form der „Anhaltenden komplexen Trauerreaktion“ (engl. Persistent Complex Bereavement Disorder). Sie findet sich in der Sektion III, die Störungsbilder enthält, die noch weiterer Forschung bedürfen, bevor sie als eigenständige Störungsbilder aufgenommen werden [13]. 


\section{Diagnosekriterien der „Anhaltenden komplexen Trauerreaktion" im DSM-5}

A. Die Person ist vom Tod eines Menschen, zu dem eine enge Beziehung bestanden hat, betroffen. B. Seit dem Todesfall tritt an mehr als der Hälfte der Tage mindestens eines der folgenden Symptome in klinisch bedeutsamer Ausprägung auf. Es besteht bei hinterbliebenen Erwachsenen für mindestens 12 Monate und bei hinterbliebenen Kindern für mindestens 6 Monaten fort:

1. Fortbestehende Sehnsucht/Verlangen nach dem Verstorbenen.

2. Intensive Sorge und emotionaler Schmerz als Reaktion auf den Todesfall.

3. Gedankliches Verhaftetsein mit dem/der Verstorbenen.

4. Übermäßige Beschäftigung mit den Umständen des Todesfalls.

C. Seit dem Todesfall tritt an mehr als der Hälfte der Tage mindestens 6 der folgenden Symptome in klinisch bedeutsamem Ausmaß auf und besteht bei hinterbliebenen Erwachsenen für mindestens 12 Monate und bei hinterbliebenen Kindern für mindestens 6 Monate fort:

\section{Durch einen Todesfall hervorgerufene Belastung}

1. Beträchtliche Schwierigkeiten, den Tod zu akzeptieren.

2. Unglaube oder emotionale Taubheit über den Verlust.

3. Schwierigkeiten, positive Erinnerungen an den Verstorbenen zuzulassen.

4. Bitterkeit oder Ärger über den Verlust.

5. Dysfunktionale Bewertungen der eigenen Person in Bezug auf den Verstorbenen oder seinen Tod (z. B. Selbstvorwürfe).
6. Übermäßiges Vermeiden von Erinnerungen an den Verlust (z. B. Vermeidung von Personen, Plätzen oder Situationen, die mit dem Verstorbenen verbunden werden).

\section{Soziale und Identitätsprobleme}

7. Der Wunsch zu sterben, um bei dem Verstorbenen zu sein.

8. Schwierigkeiten, anderen seit dem Todesfall zu vertrauen.

9. Sich seit dem Todesfall einsam oder von anderen Personen abgetrennt fühlen.

10. Das Gefühl, dass das Leben ohne den Verstorbenen sinnlos oder leer ist, oder der Glaube, dass man nicht mehr ohne den Verstorbenen funktionieren kann.

11. Verunsicherung über die eigene Rolle im Leben oder eine verminderte Wahrnehmung der eigenen Identität (z. B. das Gefühl, dass ein Teil von einem selbst mit dem Verstorbenen gestorben ist).

12. Schwierigkeiten oder Widerwillen, seit dem Verlust Interessen zu verfolgen oder Zukunftspläne zu entwickeln (z. B. Freundschaften, Aktivitäten).

D. Die Symptome verursachen in klinisch bedeutsamer Weise Leiden oder Beeinträchtigungen in sozialen, beruflichen oder anderen wichtigen Funktionsbereichen.

E. Die Trauerreaktion ist unverhältnismäßig oder nicht kongruent mit kulturellen, religiösen oder altersentsprechenden Normen.
Die anhaltende komplexe Tauerreaktion ist im DSM-5 noch nicht als eigenständiges Krankheitsbild enthalten.

ICD-11. Auch in Hinblick auf die 11. Revision der International Statistical Classification of Diseases and Related Health Problems (ICD-11) findet derzeit eine Diskussion bezüglich einer Aufnahme der komplizierten Trauer unter dem Namen „Prolongierte Trauerstörung“ (engl. Prolonged Grief Disorder) statt. Bislang ist es in der aktuellen ICD-10 nur möglich, eine pathologische Trauersymptomatik unter F43.2 (Anpassungsstörung), F43.1 (Posttraumatische Belastungsstörung), F34.1 (Dysthymie) oder unter F34.9 (anhaltende affektive Störung, nicht näher bezeichnet) zu codieren. Die bis- lang diskutierten diagnostischen Kriterien der prolongierten Trauerstörung entsprechen weitestgehend den vorgeschlagenen und empirisch validierten Kriterien von Prigerson und Mitarbeitern [11]. Im Gegensatz zu den Diagnosekriterien des DSM-5 ist das Zeitkriterium in diesem Fall deutlich kürzer (6 Monate).

\section{Erhebung der anhaltenden komplexen Trauerreaktion}

Zur Erfassung einer pathologischen Trauerreaktion ist das Inventory of Complicated Grief (ICG) das derzeit gängigste Instrument. Es wurde bereits in viele Sprachen übersetzt und findet international Anwendung 


\section{Fallbeispiel 2}

Anhaltende komplexe Trauerreaktion

Am Tage ihres 47. Geburtstags erfährt Frau Y., dass ihr Ehemann auf dem Nachhauseweg mit seinem Auto bei einem Unfall ums Leben kam. Frau Y., die in freudiger Erwartung auf ihren Mann und die anstehende Geburtstagsfeier war, ist von der Mitteilung der Polizisten vollkommen überfordert und nicht in der Lage, die Nachricht zu begreifen. Während der anschließenden Identifizierung ihres Ehemanns fühlt sie sich vollkommen von der Realität abgeschnitten, alles erscheint unwirklich. Sie hat den Eindruck in einem Film zu sein, der gleich zu Ende sein müsste. Dieser Schockzustand weicht nach wenigen Tagen einem intensiven Trauererleben und einem psychischen und physischen Schmerz, der für Frau Y. unerträglich ist. Im Wechsel dazu fühlt sie sich wie betäubt und innerlich leer. Die Beisetzung ihres Mannes erlebt Frau Y. unter der Einnahme von Benzodiazepinen. Nach der Beerdigung leidet sie unter starken Schlafstörungen, innerer Unruhe und einer intensiven und anhaltenden Traurigkeit. Sie quält sich mit Fragen, Sorgen, Ängsten, Schuldgefühlen, erlebt aber auch Gefühle von Zorn, Ärger und Verbitterung. Auch 12 Monate nach dem Verlust kann Frau Y. den Tod ihres Mannes nicht akzeptieren. Der Trauerschmerz ist nach wie vor intensiv, ihre Gedanken kreisen permanent um ihren Mann und dessen Tod und sie fühlt sich abgeschnitten vom Leben und von ihren Freunden und Verwandten. Sie hadert mit dem Schicksal, zieht sich von ihrer Umgebung zurück und sieht keinen Sinn in einer Zukunft ohne ihren Ehemann.
[14]. Von Vorteil sind zum einen die differenzialdiagnostische Abgrenzung zu Depression und Posttraumatischer Belastungsstörung (PTBS) und zum anderen die gute Vergleichbarkeit durch unterschiedliche interkulturelle Stichproben. Der Fragebogen besteht aus insgesamt 19 Items, eine deutschsprachige Evaluation liegt vor und ergab eine gute interne Konsistenz und RetestReliabilität [15]. Zusätzlich existiert eine kürzere Form des ICG, die auf Grundlage der im Jahr 2009 von Prigerson und Kollegen vorgeschlagenen Diagnosekriterien entwickelt wurde, die Prolonged Grief Scale-13 (PG-13).

\section{Prävalenz und Komorbidität}

Prävalenz. Bislang gibt es nur wenige repräsentative Untersuchungen zur Prävalenz der anhaltenden komplexen Trauerreaktion. In einer eigenen deutschen epidemiologischen Untersuchung einer repräsentativen Bevölkerungsstichprobe von 2520 Männern und Frauen im Alter von 14-95 Jahren konnte gezeigt werden, dass 3,7\% an einer komplizierten Trauersymptomatik leiden [16]. Fujisawa und Mitarbeiter fanden in ihrer bevölkerungsbasierten Studie mit 969 japanischen Männern und Frauen (40 - 79 Jahre) eine Prävalenzrate von 2,4\% [17]. In einer niederländischen bevölkerungsbasierten Kohorte mit 5741 älteren Menschen (ab 55 Jahre) lag die Prävalenz bei 4,8\%. Bei den 75 bis 85 -Jährigen waren insgesamt $7 \%$ betroffen [18]. Die höchsten Prävalenzraten finden sich innerhalb klinischer Stichproben und liegen bei Patienten mit bipolarer Erkrankung bei 24,3\% [19], bei stationären depressiven Patienten bei 18,6\% [20]. Innerhalb der Stichprobe der Trauernden weisen etwa $10 \%$ eine komplizierte Trauerreaktion auf [21].

Etwa $10 \%$ der Trauernden entwickeln eine anhaltende komplexe Trauerreaktion.

Geschlechterunterschied. Es gibt Hinweise auf geschlechtsspezifische Unterschiede, wonach Frauen häufiger betroffen zu sein scheinen als Männer [18]. Allerdings haben Frauen generell ein höheres Risiko, Verluste zu erleben, da sie zum einen eine höhere Lebenserwartung haben und zum anderen oftmals jünger sind als ihre Partner. Darüber hinaus begeben sich Frauen häufiger als Männer wegen psychischer Beschwerden in Behandlung, was ebenfalls zu einer Verzerrung führen kann.

Komorbidität. Trauernde mit einem pathologischen Trauerverlauf weisen vermehrt kardiovaskuläre Erkrankungen, Schlafstörungen und Krebserkrankungen auf [22]. Sie erkranken häufiger an komorbiden psychischen Störungen, wie z.B. Depressionen (ca. 50\%), Angststörungen (ca. 40\%) und PTBS (ca. 40\%) [17, 21]. Darüber hinaus weisen Betroffene ein erhöhtes Suizidrisiko auf $[22,23]$.

Die psychotherapeutische Behandlung der komorbiden Erkrankungen führt in der Regel zu keiner oder nur zu einer geringen Verbesserung der Symptomatik einer anhaltenden komplexen trauerreaktion.

Cave

Das Vorliegen einer anhaltenden komplexen Trauerreaktion erhöht das Suizidrisiko erheblich, sodass eine detaillierte Exploration möglicher Suizidgedanken und -handlungen erforderlich ist.

\section{Risiko- und Schutzfaktoren}

Eine anhaltende komplexe Trauerreaktion kann nach jedem Verlust auftreten, dennoch ist die Beziehung zur verstorbenen Person von Bedeutung. So ist das Risiko 
nach dem Verlust eines Kindes oder Partners höher als nach dem Verlust eines Eltern- oder Großelternteils, eines Geschwisters oder Freundes. Unter den Hinterbliebenen, die ihren Partner verloren haben, entwickeln etwa 10-20\% eine anhaltende komplexe Trauerreaktion 6-11 Monate nach dem Verlust. In einer Studie, die Eltern nach dem Verlust eines Kindes untersuchte, litten 6 Monate nach dem Verlust 59\% der Eltern unter einer Symptomatik einer anhaltenden komplexen Trauerreaktion; 12 Monate später waren es noch 38\% [24]. Auch eine ambivalente Beziehung zu der verstorbenen Person kann ein Risiko für eine Symptomatik einer anhaltenden komplexen Trauerreaktion sein. Eine bereits bestehende Traumatisierung, das Erleben früherer Verluste, eine psychische Vorerkrankung (z.B. affektive Störungen, Angststörungen, Substanzstörungen), sowie eine hohe Vulnerabilität (z.B. geringer Selbstwert, unsicherer Bindungsstil, wenig soziale Unterstützung) konnten ebenfalls als Risikofaktoren für eine Chronifizierung des Trauerprozesses identifiziert werden $[6,22]$.

Auch die konkreten Todesumstände (erwartet vs. unerwartet, gewaltsam vs. nicht gewaltsam etc.) haben einen Einfluss auf die Verarbeitung des Verlustes.

Der Verlust durch Suizid ist mit einem erhöhten Risiko für eine anhaltende komplexe Trauerreaktion verbunden. Aber auch andere plötzliche oder gewaltsame Todesumstände (z. B. Mord oder Unfall) erhöhen das Erkrankungsrisiko. Zu den weiteren Risikofaktoren gehören Alkohol- und/oder Drogenmissbrauch, geringes Einkommen, höheres Lebensalter, weibliches Geschlecht (s.o.) sowie mehrfache Verluste [12].

Persönlichkeitsfaktoren wie eine hohe wahrgenommene Kontrolle über alltägliche Aktivitäten und ein hoher Selbstwert können sich als Schutzfaktoren positiv auf den Trauerprozess auswirken. Daneben ist auch der Bindungsstil (im Sinne eines Persönlichkeitsmerkmals) von Bedeutung für die Anpassung an den Verlust eines nahestehenden Menschen. Während ein sicherer Bindungsstil ein Schutzfaktor ist, identifizierten Simon et al. einen unsicheren Bindungsstil als Risikofaktor für die Entwicklung einer anhaltenden komplexen Trauerreaktion [22].

Als eine der wichtigsten Ressourcen beim Umgang mit dem Tod einer nahe stehenden Person gilt die Unterstützung durch Angehörige und Freunde sowie das soziale Umfeld. So konnte gezeigt werden, dass Personen, die nach einem Verlust soziale Unterstützung über das Internet erhielten, weniger häufig an einer Depression, PTBS oder einer anhaltenden komplexen Trauerreaktion erkrankten [25].

Das Fehlen sozialer Unterstützung und soziale Isolation tragen zu Anpassungsschwierigkeiten bei.

\section{Differenzialdiagnostik}

Untersuchungen konnten belegen, dass die anhaltende komplexe Trauerreaktion aus einem eigenen spezifischen Symptomcluster besteht. Sie ist daher von einer Reihe anderer psychischer Erkrankungen abzugrenzen, auch wenn sich Symptome häufig überschneiden oder die Erkrankungen assoziiert miteinander auftreten können $[8,26]$.

\section{Depression}

Es bestehen enge Überschneidungen zwischen der anhaltenden komplexen Trauerreaktion und Depression, da beide ähnliche Symptome wie Traurigkeit, vermehrtes Weinen, Schlafstörungen und suizidale Gedanken umfassen. Tatsächlich tritt eine Depression häufig komorbid mit einer anhaltenden komplexen Trauerreaktion auf (s.o.), gleichzeitig werden beide Erkrankungen aber auch unabhängig voneinander diagnostiziert. So zeigten Prigerson et al., dass 46\% der Patienten mit einer komplizierten Trauer nicht die Kriterien einer Depression erfüllten [7]. Während die anhaltende komplexe Trauerreaktion einen Auslöser (den Verlust) hat und die Leitsymptome die Sehnsucht und das Verlangen nach dem verstorbenen Menschen sind, ist die Depression v.a. durch Niedergeschlagenheit, Antriebslosigkeit und Interessenverlust gekennzeichnet. Zudem ist das emotionale Erleben bei einer Depression verflacht, während es im Fall der anhaltenden komplexen Trauerreaktion eher zu rasch wechselnden emotionalen Zuständen kommt. Während eine Reihe von Untersuchungen einen deutlichen Unterschied zwischen beiden Störungsbildern zeigen, gibt es andere Studien, die eher die Gemeinsamkeiten, wie psychosoziale Beeinträchtigung, Schlafstörungen und Suizidalität, betonen [27].

Trotz Überschneidungen zwischen der anhaltenden komplexen Trauerreaktion und Depression lassen sich beide Störungsbilder voneinander abgrenzen.

\section{- Posttraumatische Belastungsstörung}

Wie bei der anhaltenden komplexen Trauerreaktion liegt auch der Posttraumatischen Belastungsstörung ein belastendes und einschneidendes Lebensereignis 
Tabelle 2

Differenzialdiagnostik von komplizierter Trauer, Depression und PTBS (nach [12]).

\begin{tabular}{|c|c|c|c|}
\hline Symptome & Komplizierte Trauer & Depression & PTBS \\
\hline \multicolumn{4}{|l|}{ Affektive Symptome } \\
\hline Traurigkeit & $\begin{array}{l}\text { stark vorhanden, auf den Verlust fokus- } \\
\text { siert, Kernsymptom }\end{array}$ & stark vorhanden, diagnostisches Kriterium & möglicherweise vorhanden \\
\hline Anhedonie & üblicherweise nicht vorhanden & $\begin{array}{l}\text { stark vorhanden und beherrschend, } \\
\text { diagnostisches Kriterium }\end{array}$ & möglicherweise vorhanden \\
\hline Angst & $\begin{array}{l}\text { möglicherweise vorhanden, fokussiert auf } \\
\text { den Verlust und die Unsicherheit ohne die } \\
\text { verstorbene Person }\end{array}$ & möglicherweise vorhanden & $\begin{array}{l}\text { stark vorhanden, fokussiert auf die } \\
\text { Angst wiederkehrender Gefahr, } \\
\text { diagnostisches Kriterium }\end{array}$ \\
\hline $\begin{array}{l}\text { Sehnsucht, Verlan- } \\
\text { gen }\end{array}$ & $\begin{array}{l}\text { stark vorhanden, häufig und intensiv, } \\
\text { Kernsymptom }\end{array}$ & üblicherweise nicht vorhanden & üblicherweise nicht vorhanden \\
\hline Schuld & $\begin{array}{l}\text { üblich, fokussiert auf Reue bezogen auf } \\
\text { die verstorbene Person }\end{array}$ & $\begin{array}{l}\text { üblicherweise vorhanden, bezogen } \\
\text { auf Gefühle von Wertlosigkeit und } \\
\text { Unwürdigkeit }\end{array}$ & $\begin{array}{l}\text { möglicherweise vorhanden, fokussiert } \\
\text { auf das traumatische Ereignis oder die } \\
\text { Folgen }\end{array}$ \\
\hline \multicolumn{4}{|c|}{ Kognitive oder behaviorale Symptome } \\
\hline $\begin{array}{l}\text { Konzentrations- } \\
\text { schwierigkeiten }\end{array}$ & $\begin{array}{l}\text { möglicherweise vorhanden, kein Kern- } \\
\text { symptom }\end{array}$ & üblich, diagnostisches Kriterium & üblich, diagnostisches Kriterium \\
\hline $\begin{array}{l}\text { gedankliche Beschäf- } \\
\text { tigung }\end{array}$ & $\begin{array}{l}\text { üblich, fokussiert auf Gedanken und } \\
\text { Erinnerungen an die verstorbene Person, } \\
\text { Kernsymptom }\end{array}$ & $\begin{array}{l}\text { möglicherweise vorhanden, fokussiert } \\
\text { auf negative Gedanken über die eigene } \\
\text { Person, andere oder die Umwelt }\end{array}$ & $\begin{array}{l}\text { negative, übertriebene, verzerrte } \\
\text { Gedanken bezogen auf das Ereignis, } \\
\text { diagnostisches Kriterium }\end{array}$ \\
\hline $\begin{array}{l}\text { wiederkehrende } \\
\text { beherrschende Bilder } \\
\text { oder Gedanken }\end{array}$ & $\begin{array}{l}\text { üblich, fokussiert auf Gedanken oder } \\
\text { Erinnerungen an die verstorbene Person }\end{array}$ & möglicherweise vorhanden & $\begin{array}{l}\text { üblich, fokussiert auf das Ereignis, } \\
\text { üblicherweise assoziiert mit Angst, } \\
\text { diagnostisches Kriterium }\end{array}$ \\
\hline $\begin{array}{l}\text { Vermeidung von } \\
\text { Erinnerungen an den } \\
\text { Verlust }\end{array}$ & $\begin{array}{l}\text { üblich, fokussiert auf Erinnerungen an } \\
\text { die Endgültigkeit des Verlustes und } \\
\text { damit verbundenes emotionales Leid }\end{array}$ & $\begin{array}{l}\text { möglicherweise vorhanden, bezogen } \\
\text { auf allgemeinen sozialen Rückzug }\end{array}$ & $\begin{array}{l}\text { üblich, fokussiert auf den Verlust eines } \\
\text { Gefühls von Sicherheit und Erinnerun- } \\
\text { gen an das Ereignis, diagnostisches } \\
\text { Kriterium }\end{array}$ \\
\hline $\begin{array}{l}\text { Suche nach Nähe zur } \\
\text { verstorbenen Person }\end{array}$ & $\begin{array}{l}\text { üblich, fokussiert auf den Wunsch, } \\
\text { der verstorbenen Person nahe zu sein }\end{array}$ & üblicherweise nicht vorhanden & üblicherweise nicht vorhanden \\
\hline
\end{tabular}

zugrunde. Bei der PTBS sind Intrusionen, Vermeidung und Übererregbarkeit die 3 zentralen diagnostischen Kriterien. Auch die Person, die an einer anhaltenden komplexen Trauerreaktion leidet, erlebt Intrusionen, die aber im Gegensatz zur PTBS nicht Erinnerungen an ein Trauma beinhalten, sondern sich auf Erinnerungen an die verstorbene Person beziehen. Zudem steht bei der anhaltenden komplexen Trauerreaktion die Trennungsangst im Vordergrund, wohingegen es bei der PTBS Ängste in Bezug auf das traumatische Ereignis bzw. die Erinnerungen an das Trauma sind. Auch das Zeitkriterium unterscheidet sich, da die Symptome der
PTBS mindestens 4 Wochen anhalten müssen, während die Diagnose einer anhaltenden komplexen Trauerreaktion nach DSM-5 erst 12 Monate nach dem Verlust gestellt werden kann. Tab. 2 fasst die wichtigsten differenzialdiagnostischen Kriterien für komplizierte Trauer, Depression und PTBS zusammen. 


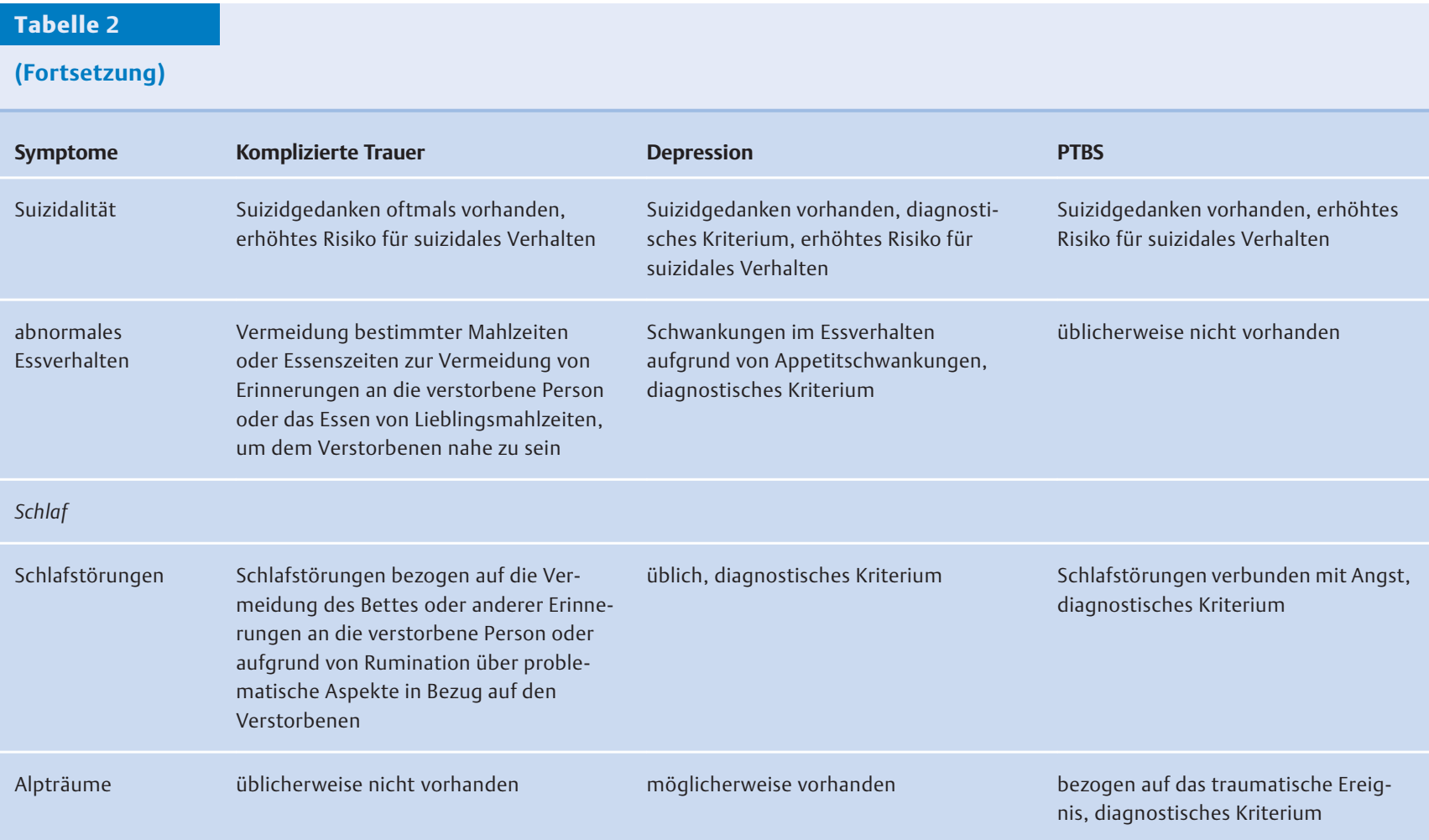

\section{Behandlung anhaltender komplexer Trauerreaktionen}

\section{Begleitung akuter Trauerprozesse}

Trauernde, die die Diagnosekriterien der anhaltenden komplexen Trauerreaktion nicht erfüllen, bedürfen keiner psychotherapeutischen Intervention. Es gibt im Gegenteil Hinweise darauf, dass diese den normalen Trauerprozess negativ beeinflussen kann [28].

\section{Die meisten Menschen bewältigen einen Verlust ohne therapeutische Hilfe.}

Es kann sinnvoll sein, den Hinterbliebenen bei Bedarf bei seiner Trauerarbeit zu unterstützen und ihn auf seinem Weg zu begleiten. Viele Trauernde erleben es als hilfreich, in einem Gespräch die Abfolge des Todes zu rekonstruieren und die Beziehung zu dem verstorbenen Menschen zu reflektieren. Auch Psychoedukation zu dem möglichen Trauerprozess und den vielfältigen dabei auftretenden Gefühlsqualitäten sowie Hinweise auf die individuelle Unterschiedlichkeit hinsichtlich dieser Prozesse und Emotionen können sinnvoll sein.

Wichtig ist eine detaillierte Exploration möglicher Suizidgedanken.

\section{Supportive Strategien zur Begleitung} akut Trauernder

- Abfolge kurz vor, während und nach dem Tod beschreiben lassen

- Beziehung zu der verstorbenen Person rekonstruieren und damit verbundene Gefühle explorieren

- Psychoedukation bzgl. oftmals mit einer Trauerreaktion einhergehendem sozialen Rückzug und Vermeidungsverhalten

- Möglichkeiten finden, auf andere Menschen zuzugehen

- ggf. Verweis auf therapeutische Hilfe (im Falle von persistierenden Symptomen, Komorbiditäten oder Suizidalität)

\section{Behandlungsansätze}

Eine anhaltende komplexe Trauerreaktion hingegen bedarf einer spezifischen therapeutischen Intervention. Derzeit bestehen verschiedene Behandlungsansätze, die jedoch nicht alle auf ihre Effektivität untersucht wurden. Vor allem kognitiv-verhaltenstherapeutische Ansätze wie von Boelen et al., Shear et al. und Rosner et al. weisen eine hohe Effektivität auf [29-31]. Die Ergebnisse dieser Studien zeigen, dass Interventionen am effektivsten sind, welche die Reduktion von vermeidendem Verhalten in Bezug auf Gedanken an 
die verstorbene Person und auf bestimmte Aktivitäten oder Orte, die mit der verstorbenen Person in Verbindung gebracht werden, zum Ziel haben. Auch das Bearbeiten von Intrusionen, Schuld- und Schamgefühlen sowie von dysfunktionalen Gedanken ist zentraler Bestandteil kognitiv-verhaltenstherapeutisch ausgerichteter Therapien für pathologische Trauerverläufe.

\section{Komplizierte Trauertherapie}

Ein hinsichtlich seiner Effektivität bislang gut untersuchtes Behandlungskonzept ist die komplizierte Trauertherapie von Shear und Mitarbeitern, die Ansätze der kognitiven Verhaltenstherapie mit Aspekten der interpersonellen Therapie kombiniert [30]. Ziel ist es, Komplikationen, die den Trauerverlauf blockieren, zu identifizieren und zu bearbeiten, um den normalen Trauerprozess zu unterstützen. Im Verlauf von 16 wöchentlich stattfindenden Sitzungen wird der Fokus sowohl auf das Wiedererlangen einer normalen und gesunden Funktionsfähigkeit gelegt (bewältigungsorientierte Techniken) sowie auf die Möglichkeit für den Trauernden, einen Weg für den Umgang mit dem Verlust zu finden und an die verstorbene Person zu denken, ohne dass intensive Gefühle von Wut, Schuld oder Angst vorherrschend sind (verlustorientierte Techniken). Bestandteile dieser Therapie sind:

- Expositionsübungen (Konfrontation mit bisher vermiedenen trauerspezifischen Stimuli)

- das Führen eines Trauertagebuchs

- das wiederholte Erzählen der Geschichte des Verlustes

- die Einbeziehung einer aktuellen Bezugsperson

\section{- Internetbasierte Therapie}

In den letzten Jahren haben internetbasierte Interventionsprogramme an Bedeutung gewonnen. Verschiedene Studien belegen die Wirksamkeit dieses Ansatzes für eine Vielzahl psychischer Erkrankungen. Darauf aufbauend entwickelten Wagner und Mitarbeiter ein internetbasiertes Therapieprogramm für komplizierte Trauer, welches auf einem kognitiv-verhaltenstherapeutischen Ansatz beruht [32]. Teilnehmer mit einer komplizierten Trauersymptomatik wiesen nach Abschluss dieser 5-wöchigen Intervention deutlich weniger Trauersymptome und eine reduzierte depressive Symptomatik auf im Vergleich zu einer Kontrollgruppe. Die Effekte waren 1,5 Jahre nach Beendigung der Therapie noch nachweisbar.

Effekte internetbasierter Programme sind auch nach über einem Jahr noch nachweisbar.
Die 7 Hauptkomponenten der

Behandlung komplizierter Trauer

1. Beschreibung des Verlustes und des Trauererlebens, Erfassung komplizierter Trauersymptome und deren Auswirkungen, Erläuterung der Behandlung und der Vorgehensweise

2. Selbstregulation stärken, Selbstbeobachtung und Reflektion, Neubewertung beunruhigender Gedanken und Überzeugungen, „Dosierung“ von emotionalem Schmerz durch Konfrontieren und Beiseitelegen

3. Verbindungen schaffen, Strategien zum Etablieren von bedeutungsvollen Beziehungen zu anderen, Teilen von Schmerz, Akzeptanz von Hilfe durch andere

4. Suchen und Setzen persönlicher Ziele und Aktivitäten, die Hoffnung, Begeisterung und andere positive Emotionen auslösen, ein Gefühl für mögliches zukünftiges Glück schaffen

5. Entwicklung von Strategien zur Konfrontation oder zum Überdenken vermiedener Situationen

6. Erzählung und Reflektion der Geschichte der verstorbenen Person mit dem Ziel einer angemessenen Beschreibung; üben den Schmerz zu konfrontieren und beiseite zu legen

7. Überdenken positiver und negativer Erinnerungen an die verstorbene Person, Beschreibung einer imaginierten Konversation mit dem Toten

(nach [12])

In einer eigenen internetbasierten Studie, die sich an Eltern nach dem Verlust eines Kindes in der Schwangerschaft richtete, wurden diese aufgefordert, 10 Schreibaufgaben zu verfassen, welche die folgenden Bereiche umfassten:

- Selbstkonfrontation

- kognitives Wiedererleben bzw. kognitive Umstrukturierung

- Social Sharing (andere am Erlebten teilhaben lassen) [33]

Nach Abschluss der ebenfalls 5-wöchigen Behandlung wiesen die Teilnehmer eine deutliche Reduzierung der komplizierten Trauersymptomatik, des traumatischen Erlebens und der depressiven Symptomatik auf. Diese Symptomreduzierung war statistisch signifikant höher als als bei einer Wartelistenkontrollgruppe. Diese Verbesserung auf allen Symptomebenen war auch 3 bzw. 12 Monate nach Behandlungsende nachweisbar. 


\section{Psychopharmakotherapie}

Auch wenn die Studienlage zur Pharmakotherapie bei pathologischen Trauerverläufen momentan uneinheitlich ist, wird die Gabe von Antidepressiva in der pharmakologischen Behandlung der anhaltenden komplexen Trauerreaktion diskutiert. Reynolds et al. sowie Pasternak et al. fanden jedoch lediglich eine Reduktion der depressiven Symptomatik durch die Gabe einer antidepressiven Medikation, nicht jedoch eine Reduktion der Symptome der anhaltenden komplexen Trauerreaktion [34, 35]. Andere Studien hingegen konnten einen Effekt von Antidepressiva auf die Symptomatik der komplizierten Trauer nachweisen, auch wenn es sich dabei lediglich um einen minimalen bis moderaten Effekt handelte [36, 37]. Zwei Open-Label-Studien weisen zudem darauf hin, dass der selektive SerotoninWiederaufnahmehemmer Escitalopram effektiv auf die Symptomatik der komplizierten Trauer wirkt [38, 39].

Weiterhin konnte nachgewiesen werden, dass die Abbruchrate der psychotherapeutischen Behandlung bei komplizierter Trauer niedriger ist bei Patienten mit antidepressiver Medikation im Vergleich zu jenen ohne regelmäßige Einnahme von Antidepressiva [22].

\section{Fazit für die Praxis}

Die psychotherapeutische Behandlung der anhaltenden komplexen Trauerreaktion ist das Mittel der Wahl.

\section{Fazit und Perspektiven}

Trauer per se ist kein pathologisches Phänomen, sondern ein ubiquitärer Vorgang. Ein Großteil der Verluste wird von den Hinterbliebenen im Rahmen einer normalen Trauerreaktion angemessen bewältigt. Bei Nichtbewältigung können psychische und/oder physische Erkrankungen die Folge sein, die jedoch häufig nicht mit dem unbewältigten Trauerprozess in Zusammenhang gebracht werden.

Bei einem Teil der Trauernden mündet der Trauerprozess in eine anhaltende komplexe Trauerreaktion, die einer diagnostischen und differenzialdiagnostischen Abklärung und einer entsprechenden Behandlung bedarf.

Während normal Trauernde in der Regel keine therapeutische Intervention benötigen, existieren aktuell verschiedene Behandlungsansätze, die sich an Menschen mit einem pathologischen Trauerverlauf richten. Mit ihrer Hilfe kann eine deutliche Verbesserung der Symptomatik erzielt werden. So gilt es, den klinischen Blick für diese spezielle Gruppe von Trauernden zu schärfen und davon Betroffene zu identifizieren, um sie dabei zu unterstützen, ihren Trauerprozess zu bewältigen und sich wieder dem Leben zuzuwenden.

\section{Kernaussagen}

Der normale Trauerprozess wird durch eine Vielzahl individueller und kultureller Faktoren beeinflusst. Psychische oder physische Belastungen und Symptome können Teil dieses Prozesses sein, sich infolge des Verlustes eines nahestehenden Menschen, aber auch als eigenständige Erkrankung manifestieren. Etwa 10\% der Trauernden können den Trauerprozess nicht abschließen und entwickeln eine anhaltende komplexe Trauerreaktion. Diese ist wissenschaftlichen Untersuchungen zufolge als eigenständiges Krankheitsbild anzusehen, für das empirisch validierte Kriterien bestehen. Es ist abgrenzbar von anderen psychischen Erkrankungen, die häufig mit einer anhaltenden komplexen Trauerreaktion komorbid auftreten (z. B. Depression, Angststörungen oder PTBS). Risikofaktoren für die Entstehung einer anhaltenden komplexen
Trauerreaktion umfassen u. a. mehrfache Verluste, traumatische Todesumstände, eine bereits bestehende Traumatisierung, eine psychische Vorerkrankung sowie eine ambivalente Beziehung zu der verstorbenen Person. Während der normale Trauerprozess in der Regel keine spezifische therapeutische Behandlung benötigt, gibt es spezielle psychotherapeutische Interventionen für Hinterbliebene mit einem pathologischen Trauerverlauf. Vor allem für verhaltenstherapeutisch basierte Konzepte wurde eine hohe Effektivität nachgewiesen. Sie können den Betroffenen dabei helfen, den Trauerprozess zu bewältigen. Im Falle einer schwerwiegenden komorbiden depressiven Erkrankung kann ergänzend zur psychotherapeutischen Intervention eine medikamentöse Behandlung in Betracht gezogen werden. 


\section{Über die Autorinnen}

\section{Anette Kersting}

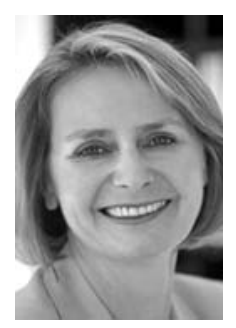

Prof. Dr. med. Studium der Humanmedizin in Hannover, Facharztweiterbildung Psychosomatische Medizin und Psychotherapie, Zusatztitel Psychoanalyse, Weiterbildungen in Systemischer Therapie, Traumatherapie, IPT. Wissenschaftliche Mitarbeiterin in der Klinik für Psychosomatik und Psychotherapie der Medizinischen Hochschule Hannover sowie der Christian-Albrechts-Universität zu Kiel, Oberärztin und Leiterin des Bereichs Psychotherapie an der Klinik und Poliklinik für Psychiatrie und Psychotherapie des Universitätsklinikums Münster. Seit 2010 Direktorin der Klinik und Poliklinik für Psychosomatische Medizin und Psychotherapie am Universitätsklinikum Leipzig. Lehrstuhlinhaberin Psychosomatische Medizin und Psychotherapie der Medizinischen Fakultät Leipzig. Forschungsschwerpunkte: Trauer, Psychotherapieforschung, Essstörungen.

\section{Jana Steinig}

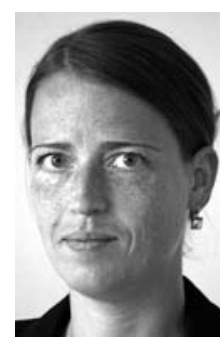

Dr. phil. Studium der Psychologie und Promotion an der Universität Bremen. Seit 2010 wissenschaftliche Mitarbeiterin an der Klinik und Poliklinik für Psychosomatische Medizin und Psychotherapie am Universitätsklinikum Leipzig. Forschungsschwerpunkte: Internetbasierte Therapie (u.a. für trauernde Eltern nach Verlust eines Kindes in der Schwangerschaft oder für Patienten mit Binge-Eating-Störung). Seit 2010 in psychotherapeutischer Ausbildung für die Verfahren Tiefenpsychologie und Psychoanalyse am Sächsischen Institut für Psychoanalyse und Psychotherapie - Therese Benedek (SPP).

Interessenkonflikt: Beide Autorinnen geben an, dass in Bezug auf die Veröffentlichung kein Interessenkonflikt besteht.

\section{Korrespondenzadresse}

Dr. phil. Jana Steinig, Dipl.-Psych.

Universitätsklinikum Leipzig

Department Psychische Gesundheit

Klinik und Poliklinik für Psychosomatische Medizin und

Psychotherapie

Semmelweisstraße 10

04103 Leipzig

E-Mail: Jana.Steinig@medizin.uni-leipzig.de

Verantwortlicher Herausgeber für diesen Beitrag:

Prof. Dr. Fritz Hohagen, Lübeck

\section{Literatur}

1 Kast V. Natürliche Trauer - komplizierte Trauer. PsychotherWiss 2011; 1: 94-101

2 Kübler-Ross E. On death and dying. New York: Macmillan; 1969

3 Stroebe M, Schut $\mathrm{H}$. The dual process model of coping with bereavement: rationale and description. Death Stud 1999; 23 : $197-224$

4 Bonanno GA, Kaltman S. The varieties of grief experience. Clin Psychol Rev 2001; 21: 705-734

5 Kersting A, Kroker K, Steinhard J et al. Complicated grief after traumatic loss: a 14-month follow up study. Eur Arch Psychiatry Clin Neurosci 2007; 257: 437-443

6 Stroebe M, Schut H, Stroebe W. Health outcomes of bereavement. Lancet 2007; 370: 1960 - 1973

7 Prigerson HG, Frank E, Kasl SV et al. Complicated grief and bereavement-related depression as distinct disorders: preliminary empirical validation in elderly bereaved spouses. Am J Psychiatry 1995; 152: $22-30$

8 Boelen PA, van den Bout J. Complicated grief, depression, and anxiety as distinct postloss syndromes: a confirmatory factor analysis study. Am J Psychiatry 2005; 162: 2175 - 2177

9 Barnes JB, Dickstein BD, Maguen S et al. The distinctiveness of prolonged grief and posttraumatic stress disorder in adults bereaved by the attacks of September 11th. J Affect Disord 2012; 136: 366-369

10 Horowitz M], Siegel B, Holen A et al. Diagnostic criteria for complicated grief disorder. Am J Psychiatry 1997; 154: 904 910

11 Prigerson HG, Horowitz MJ, Jacobs SC et al. Prolonged grief disorder: psychometric validation of criteria proposed for DSM-V and ICD-11. PLoS Med 2009; 6: e1000121

12 Shear MK. Clinical practice. Complicated grief. N Engl J Med 2015; 372: $153-160$

13 American Psychiatric Association. Diagnostic and statistical manual of mental disorders: DSM-V. 5th ed. Washington, DC: American Psychiatric Association; 2013

14 Prigerson HG, Maciejewski PK, Reynolds CF et al. Inventory of complicated grief: a scale to measure maladaptive symptoms of loss. Psychiatry Res 1995; 59: 65-79 
15 Lumbeck G, Brandstätter M, Geissner E. Erstvalidierung der deutschen Version des „Inventory of Complicated Grief” (ICGD). Z Für Klin Psychol Psychother 2012; 41: 243 - 248

16 Kersting A, Brähler E, Glaesmer $\mathrm{H}$ et al. Prevalence of complicated grief in a representative population-based sample. J Affect Disord 2011; 131: 339-343

17 Fujisawa D, Miyashita M, Nakajima S et al. Prevalence and determinants of complicated grief in general population. J Affect Disord 2010; 127: 352 - 358

18 Newson RS, Boelen PA, Hek K et al. The prevalence and characteristics of complicated grief in older adults. J Affect Disord 2011; 132: $231-238$

19 Simon NM, Shear KM, Thompson EH et al. The prevalence and correlates of psychiatric comorbidity in individuals with complicated grief. Compr Psychiatry 2007; 48: 395 - 399

20 Kersting A, Kroker K, Horstmann J et al. Complicated grief in patients with unipolar depression. J Affect Disord 2009; 118 : $201-204$

21 Shear MK, Simon N, Wall M et al. Complicated grief and related bereavement issues for DSM-5. Depress Anxiety 2011; 28: $103-117$

22 Simon NM. Treating complicated grief. J Am Med Assoc 2013; 310: $416-423$

23 Young IT, Iglewicz A, Glorioso D et al. Suicide bereavement and complicated grief. Dialogues Clin Neurosci 2012; 14 : $177-186$

24 Meert KL, Shear K, Newth CJL et al. Follow-Up Study of complicated grief among parents eighteen months after a child's death in the pediatric intensive care unit. J Palliat Med 2011; 14: $207-214$

25 Vanderwerker LC, Prigerson HG. Social support and technological connectedness as protective factors in bereavement. J Loss Trauma 2004; 9: 45-57

26 Lichtenthal WG, Cruess DG, Prigerson HG. A case for establishing complicated grief as a distinct mental disorder in DSMV. Clin Psychol Rev 2004; 24: 637-662

27 Zisook S, Shear K, Kendler KS. Validity of the bereavement exclusion criterion for the diagnosis of major depressive episode. World Psychiatry 2007; 6: 102 - 107

28 Currier JM, Neimeyer RA, Berman JS. The effectiveness of psychotherapeutic interventions for bereaved persons: a comprehensive quantitative review. Psychol Bull 2008; 134: $648-661$

29 Boelen PA, de Keijser J, van den Hout MA et al. Treatment of complicated grief: a comparison between cognitive-behavioral therapy and supportive counseling. J Consult Clin Psychol 2007; 75: $277-284$

30 Shear K, Frank E, Houck PR et al. Treatment of complicated grief: a randomized controlled trial. J Am Med Assoc 2005; 93 : $2601-2608$

31 Rosner R, Bartl H, Pfoh G et al. Efficacy of an integrative CBT for prolonged grief disorder: A long-term follow-up. J Affect Disord 2015; 183: 106-112

32 Wagner B, Knaevelsrud C, Maercker A. Internet-based cognitive-behavioral therapy for complicated grief: a randomized controlled trial. Death Stud 2006; 30: 429-453

33 Kersting A, Dölemeyer R, Steinig J et al. Brief internet-based intervention reduces posttraumatic stress and prolonged grief in parents after the loss of a child during pregnancy: a randomized controlled trial. Psychother Psychosom 2013; 82: $372-381$

34 Reynolds CF 3rd, Miller MD, Pasternak RE et al. Treatment of bereavement-related major depressive episodes in later life: a controlled study of acute and continuation treatment with nortriptyline and interpersonal psychotherapy. Am J Psychiatry 1999; 156: $202-208$

35 Pasternak RE, Reynolds CF 3rd, Schlernitzauer M et al. Acute open-trial nortriptyline therapy of bereavement-related depression in late life. J Clin Psychiatry 1991; 52: 307 - 310

36 Zygmont M, Prigerson HG, Houck PR et al. A post hoc comparison of paroxetine and nortriptyline for symptoms of traumatic grief. J Clin Psychiatry 1998; 59: 241 - 245

37 Zisook S, Shuchter SR, Pedrelli P et al. Bupropion sustained release for bereavement: results of an open trial. J Clin Psychiatry 2001; 62: $227-230$

38 Simon NM, Thompson EH, Pollack MH et al. Complicated grief: a case series using escitalopram. Am J Psychiatry 2007; 164: 1760 - 1761

39 Hensley PL, Slonimski CK, Uhlenhuth EH et al. Escitalopram: an open-label study of bereavement-related depression and grief. J Affect Disord 2009; 113: $142-149$ 


\section{CME-Fragen}

\section{CME.thieme.de}

\section{CME-Teilnahme}

- Viel Erfolg bei lhrer CME-Teilnahme unter http://cme.thieme.de

- Diese Fortbildungseinheit ist 12 Monate online für eine CME-Teilnahme verfügbar.

- Sollten Sie Fragen zur Online-Teilnahme haben, unter http://cme.thieme.de/hilfe finden Sie eine ausführliche Anleitung.

1

Welche der nachfolgenden Aussagen zur normalen Trauer ist falsch?
A Es gibt keine klar definierte Grenze zwischen normaler und pathologischer Trauer.

B Fehlende Trauer ist eine Variante des Bewältigungsprozesses.

C Der normale Trauerprozess lässt sich in eine feste Abfolge von einzelnen Stufen einteilen.

D Trauer per se ist kein pathologisches Phänomen.

E Phasenmodelle zum normalen Trauerverlauf berücksichtigen individuelle und kulturelle Unterschiede nur unzureichend.
Welche Aussage über das duale Prozessmodell ist falsch?
A Hauptziel ist die Loslösung von der verstorbenen Person.
B Kernaspekt ist das dynamische Oszillieren zwischen 2 Formen der Trauerbewältigung.
C Verlustorientierte Verarbeitungsprozesse sind gleichwertig gegenüber wiederherstellungsorientierten Verarbeitungsprozessen.
D Trauerarbeit gehört zur verlustorientierten Bewältigung. E Die wiederherstellungsorientierte Bewältigung fokussiert sich auf die Orientierung auf neue
Lebensziele.

3

Wie viel Prozent der Trauernden entwickeln eine komplizierte Trauer?
A ca. $5 \%$
B ca. $10 \%$
C $15-25 \%$
D $>30 \%$
E ca. $40 \%$

4

Welche Aussage zur Aufnahme der anhaltenden komplexen Trauerreaktion in die diagnostischen Klassifikationssysteme ist richtig?
A Die anhaltende komplexe Trauerreaktion wird sowohl im aktuellen DSM-5 als auch in der geplanten ICD-11 als eigenständige Erkrankung klassifiziert.

B Die anhaltende komplexe Trauerreaktion ist in der aktuellen DSM-5 als eigenständige Erkrankung klassifiziert.

C Die Aufnahme der anhaltenden komplexen Trauerreaktion in die Neuauflage der ICD wird derzeit noch diskutiert

D In der aktuellen DSM-5 ist die anhaltende komplexe Trauerreaktion eine Unterform der Anpassungsstörung.

E Die anhaltende komplexe Trauerreaktion ist in der aktuellen ICD-10 als eigenständige Erkrankung klassifiziert mit eindeutig nachgewiesenen diagnostischen Kriterien.

\section{5}

Welche Aussage zu möglichen Risikofaktoren für eine anhaltende komplexe Trauerreaktion ist richtig?
A Eine anhaltende komplexe Trauerreaktion ist umso wahrscheinlicher, je mehr die verstorbene Person geliebt wurde.

B Das Risiko einer anhaltenden komplexen Trauerreaktion ist nach einem Verlust eines Kindes ebenso hoch wie nach dem Verlust eines Partners.

C Eine ambivalente Beziehung zu der verstorbenen Person erhöht das Risiko einer anhaltenden komplexen Trauerreaktion.

D Das Erleben früherer Verluste ist kein Risikofaktor für eine anhaltende komplexe Trauerreaktion, da der Prozess des Trauerns dem Hinterbliebenen bereits bekannt ist.

E Frauen und Männer haben die gleiche Wahrscheinlichkeit, eine anhaltende komplexe Trauerreaktion zu entwickeln. 


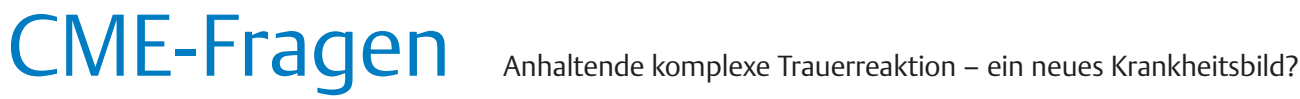

\section{6}

Wie viel Prozent der Menschen, die eine anhaltende komplexe Trauerreaktion aufweisen, leiden an einer komorbiden Depression?
A $5-10 \%$
B ca. $25 \%$
C $30-40 \%$
D ca. $50 \%$
E $>50 \%$

7

Welche Aussage zur Behandlung von Trauer ist richtig?
A Der Trauerprozess nach einem Verlust sollte in jedem Fall therapeutisch begleitet werden, um einer pathologischen Trauerreaktion vorzubeugen.

B Es sollten v. a. eventuelle komorbide Erkrankungen, wie z. B. eine Depression, behandelt werden, dann bessert sich die Trauersymptomatik von selbst.

C Interventionen, die sich pauschal an alle Trauernden richten, sind nicht nötig und könnten den normalen Trauerverlauf evtl. sogar negativ beeinflussen.

D Eine medikamentöse Behandlung ist das Mittel der Wahl.

E Der Hinterbliebene sollte in seiner Trauer möglichst ungestört bleiben, um eventuelle Komplikationen im Trauerverlauf zu vermeiden.

\section{8}

Welche Aussage zur psychotherapeutischen Behandlung von pathologischer Trauer ist falsch?
A Das Behandlungskonzept für komplizierte Trauer nach Shear und Mitarbeitern ist auf einen Zeitraum von 6 Monaten angelegt.

B Intrusionen, Schuld- und Schamgefühle sowie dysfunktionale Gedanken sollten im Rahmen der Therapie fokussiert werden.

C Das Behandlungskonzept für komplizierte Trauer nach Shear und Mitarbeitern kombiniert Aspekte der kognitiven Verhaltenstherapie mit Ansätzen der interpersonellen Therapie.

D Vor allem kognitiv-verhaltenstherapeutisch ausgerichtete Behandlungsansätze weisen eine hohe Effektivität auf.

E Es sind v. a. jene Interventionen effektiv, die die Reduktion von vermeidendem Verhalten fokussieren.

9

Was zählt nicht zu den Hauptkomponenten der Behandlung komplizierter Trauer nach Shear?
A Beziehungen zu anderen aufbauen

B imaginierte Konversation mit der verstorbenen Person

C das Konfrontieren und Teilen von schmerzhaften Gedanken, Emotionen und Erinnerungen

D das Entfernen von Gegenständen aus dem näheren Umfeld des Trauernden, die an die verstorbene Person erinnern

E das Planen und Umsetzen von positiven Aktivitäten

\section{0}

Welche Aussage zur Psychopharmakotherapie der anhaltenden komplexen Trauerreaktion ist falsch?
A Eine medikamentöse Begleittherapie ist zusätzlich zu einer psychotherapeutischen Behandlung immer angezeigt.

B Die Abbruchrate psychotherapeutischer Behandlung der komplizierten Trauer ist niedriger bei Patienten mit antidepressiver Medikation als bei Patienten ohne Medikation.

C Die Forschungsergebnisse zu Pharmakotherapie bei pathologischem Trauerverlauf sind uneinheitlich.

D Bei einer ausgeprägten komorbiden depressiven Erkrankung sollte die psychotherapeutische Behandlung um eine zusätzliche Medikation mit Antidepressiva ergänzt werden.

E Einige Studien fanden Hinweise darauf, dass sich lediglich die depressive Symptomatik durch die Gabe einer antidepressiven Medikation reduziert - nicht die Symptomatik der anhaltenden komplexen Trauerreaktion. 